\title{
Signal Space Separation Beamformer
}

\author{
Jiri Vrba $\cdot$ Samu Taulu $\cdot$ Jukka Nenonen $\cdot$ \\ Antti Ahonen
}

Received: 7 July 2009/ Accepted: 7 November 2009/Published online: 27 November 2009

(C) The Author(s) 2009. This article is published with open access at Springerlink.com

\begin{abstract}
We have combined Signal Space Separation and beamformers (SSS beamformer). The SSS beamformer was tested by simulation in the presence of simulated brain noise. The SSS beamformer performs at least as well as the conventional beamformer, provided that the expansion order is sufficiently high. For beamformer outputs which depend on power or power difference normalized by the projected noise, the spatial resolution of the SSS beamformer is significantly better than that of the conventional beamformers if the sources are deeper, and about the same as that of the conventional beamformer when the sources are superficial. For beamformer outputs which depend on the ratio of powers, the spatial resolutions of the SSS and conventional beamfomers are the same. The sensor noise covariance matrix in the SSS basis is non-diagonal. The SSS beamformers with diagonalized noise covariance matrix exhibit better spatial resolution than that with nondiagonal noise covariance matrix. The SSS beamformers are computationally more efficient than the conventional beamformers.
\end{abstract}

Keywords Beamformers - Signal space separation . Magnetoencephalography $\cdot$ MEG $\cdot$ Signal processing

This is one of several papers published together in Brain Topography on the "Special Topic: Cortical Network Analysis with EEG/MEG".

J. Vrba $\cdot$ S. Taulu $\cdot$ J. Nenonen $\cdot$ A. Ahonen

Elekta Oy, Helsinki, Finland

J. Vrba $(\bowtie)$

Coquitlam, BC V3C 5A8, Canada

e-mail: jvrba@shaw.ca

\section{Introduction}

Beamformers have an important role in non-parametric analysis of the source activity underlying magnetoencephalography (MEG) data (Van Veen et al. 1997; Robinson and Vrba 1998; Gross et al. 2001; Sekihara et al. 2004). Beamformers are spatial filters designed to extract electrical activity from target brain location while suppressing contributions originating outside the target. Beamformer weights are based on data and they do not require a priori assumptions about the number of active sources, solutions are analytical (there is no need for global minimum searches of a cost function), estimates for each voxel can be made independent of other voxels, there is no tendency of source drift to surface (as in minimum norm based approaches), and the beamformers can be used to image spectral power which is not necessarily phase locked to stimulus (Barnes and Hillebrand 2003). A disadvantage of beamformers is that they tend to suppress spatially separate yet covariant sources, and in addition, the spatial resolution at a particular source space region depends on the data.

Biological magnetic fields are measured by multichannel sensors. High spatial frequencies of the magnetic fields decay rapidly with increasing source depth and only relatively low spatial frequencies exceed the sensor noise levels, resulting in less than 200 degrees of freedom of the measurable MEG signals (Ahonen et al. 1993). The modern MEG devices employ over 250 channels and spatially oversample the signal. It was proposed (Taulu and Kajola 2005) to express the magnetic field by a truncated basis function expansion, which allows description of the magnetic field by fewer basis functions than the number of physical channels. The method is called signal space separation (SSS). The magnetic field can be represented as a 
combination of two separate expansions: one corresponding to fields originating from the volume of interest (internal terms) and the second to fields arising outside the volume of interest (external terms). Elimination of the external terms from the expansion allows for suppression of the external interference without distorting the measured MEG signals.

After the introduction of the SSS method it was proposed to combine the beamforming and SSS approaches into an SSS beamformer (Nenonen and Taulu 2005). This approach utilizes harmonic function amplitudes and vector spherical harmonic leadfields obtained from the signal space separation method instead of the measured sensor values. Initially, it was thought that the method would provide effective data compression and improved computational efficiency-instead of 306 measured values at a given time the new method would typically need only about 100 harmonic amplitudes. However, it was found that in addition to reducing computational load, the method also improves spatial resolution of certain types of beamformers.

In this work we compare spatial resolution of the SSS and conventional beamformers by simulations. Other parameters, e.g., localization accuracy, are comparable for the two beamformer types and are not discussed in the paper.

\section{Materials and Methods}

This section describes the SSS formulation, conventional and SSS beamformers, the beamformer constructs used in the work, and simulation parameters.

\section{Theoretical Background}

Measurement at time $t$ is a column vector $\mathbf{m}(t)$ with dimensions $M \times 1$, where $M$ is the number of $\mathrm{MEG}$ channels.

Because the MEG sensors are located in a source-free volume, the magnetic field, $\mathbf{B}$, can be expressed as a gradient of a scalar potential, $\Psi, \mathbf{B}=-\nabla \Psi$, which is a solution of Laplace equation, $\nabla^{2} \Psi=0$. Such a solution can be represented as a linear combination of basis functions, e.g., spherical harmonics, as

$\Psi(\mathbf{r})=\sum_{n=0}^{\infty} \sum_{m=-n}^{n} a_{n m} \frac{Y_{n m}(\theta, \varphi)}{r^{n+1}}+\sum_{n=0}^{\infty} \sum_{m=-n}^{n} b_{n m} r^{n} Y_{n m}(\theta, \varphi)$

where $Y_{n m}$ are spherical harmonic functions, $\theta$ and $\varphi$ denote spherical angles, $r=|\boldsymbol{r}|$ is the distance from the expansion center, and $a_{n m}$ and $b_{n m}$ are expansion coefficients. The first term on the right-hand side of Eq. 1 diverges at the origin and it represents sources within the sensor shell; the second term diverges at infinity and corresponds to sources outside the sensor shell. Contributions of the internal and external sources can be separated and the external terms can be discarded to reduce the environmental noise.

The MEG is measured by SQUID sensors which typically consist of several sensing coils. Magnetic fields for a given sensor at all coil positions, as expressed by gradient of Eq. 1, are combined. Then, the sensor array measurement can be expressed in terms of spherical harmonics as $\mathbf{m}(t) \approx \mathbf{S X}(t)$, where the matrix $\mathbf{S}=\left[\mathbf{S}_{\text {in }} \mathbf{S}_{\text {ext }}\right]$ contains the basis vectors and has dimension $M \times D, D$ is the number of the basis vectors $(D<M)$, and $\mathbf{X}(t)$ is time dependent column vector of the basis vector amplitudes. The $\mathbf{S}_{\text {in }}$ contains internal and $\mathbf{S}_{\text {ext }}$ external expansion terms (see Eq. 1). The " $\approx "$ sign is used because the expansion is truncated at $n_{\text {int }}$ internal terms and $n_{\text {ext }}$ external terms. The time dependent coefficients $\mathbf{X}(t)$ can be estimated as $\tilde{\mathbf{X}}(t) \approx \mathbf{S}^{+} \mathbf{m}(t)$, where $\mathbf{S}^{+}$is pseudoinverse of $\mathbf{S}$. The MEG measurement with external interference filtered out can be obtained as $\tilde{\mathbf{m}}(t)=\mathbf{V m}(t)$, where the matrix $\mathbf{V}=\mathbf{S}_{\text {in }} \mathbf{P S}^{+}$ and $\mathbf{P}=[\mathbf{I}$ 0] (Taulu and Kajola 2005).

Only the scalar beamformers will be discussed. Equations for beamformer are well known (e.g., Sekihara et al. 2004) and general forms of the power and power normalized by noise (pseudo- $Z^{2}$ ) are:

$$
P(\mathbf{r}, \boldsymbol{\eta})=\frac{1}{\boldsymbol{\Phi}^{T}(\mathbf{r}, \boldsymbol{\eta}) \mathbf{C}_{M}^{-1} \boldsymbol{\Phi}(\mathbf{r}, \boldsymbol{\eta})}
$$

$Z^{2}(\mathbf{r}, \boldsymbol{\eta})=\frac{\boldsymbol{\Phi}^{T}(\mathbf{r}, \boldsymbol{\eta}) \mathbf{C}_{M}^{-1} \boldsymbol{\Phi}(\mathbf{r}, \boldsymbol{\eta})}{\boldsymbol{\Phi}^{T}(\mathbf{r}, \boldsymbol{\eta}) \mathbf{C}_{M}^{-1} \mathbf{C}_{N} \mathbf{C}_{M}^{-1} \boldsymbol{\Phi}(\mathbf{r}, \boldsymbol{\eta})}$

where $\mathbf{r}$ is the vector of source position, and $\boldsymbol{\eta}$ is $3 \times 1$ (or $2 \times 1)$ source orientation vector. For conventional beamformers, $\boldsymbol{\Phi}(\mathbf{r}, \boldsymbol{\eta})=\mathbf{L}(\mathbf{r}, \boldsymbol{\eta})$ is $M \times 1$ lead field matrix, $\mathbf{C}_{M}=\mathbf{C}_{m}$ is covariance matrix of the measurement with dimension $M \times M$, and $\mathbf{C}_{N}=\mathbf{C}_{v}$ is the noise covariance matrix computed from instrumental noise time courses, $v(t)$. The lead field matrix can be separated into the position and orientation parts $\mathbf{L}(\mathbf{r}, \boldsymbol{\eta})=\mathbf{L}(\mathbf{r}) \boldsymbol{\eta}(\mathbf{r})$ and the source orientation which maximizes either the $P$ or $Z^{2}$ can be found by procedure described in (Sekihara et al. 2004).

For the SSS beamformer, $\mathbf{C}_{M}=\mathbf{C}_{x}$ is the covariance matrix of the time dependent expansion coefficients $\mathbf{X}(t)$ with dimension $D \times D$ and is related to the covariance matrix of measurement by $\mathbf{C}_{x}=\mathbf{S}^{+} \mathbf{C}_{m} \mathbf{S}^{+T}, \mathbf{C}_{N}=\mathbf{C}_{x v}$ is the noise covariance matrix computed from instrumental noise transformed into the SSS basis and is related to the conventional noise covariance matrix by $\mathbf{C}_{x v}=\mathbf{S}^{+} \mathbf{C}_{v} \mathbf{S}^{+T}$, and $\boldsymbol{\Phi}(\mathbf{r}, \boldsymbol{\eta})=\boldsymbol{\Gamma}(\mathbf{r}, \boldsymbol{\eta})$ is $D \times 1 \mathrm{SSS}$ lead field matrix. The $\boldsymbol{\Gamma}$ is related to $\mathbf{L}$ by $\boldsymbol{\Gamma}(\mathbf{r}, \boldsymbol{\eta})=\mathbf{S}^{+} \mathbf{L}(\mathbf{r}, \boldsymbol{\eta})$. The $\boldsymbol{\Gamma}$ can also be decomposed into the position and orientation parts, and 
source orientation which maximizes $P$ or $Z^{2}$ can be found the same way as for the conventional beamformers. To compute the SSS beamformer, the data and noise are first transformed into the SSS basis and then the covariance matrices $\mathrm{C}_{x}$ and $\mathrm{C}_{x v}$ are computed. Instead of directly computing the lead field matrix in the SSS basis, we have used the relationship $\boldsymbol{\Gamma}=\mathbf{S}^{+} \mathbf{L}$. The SSS noise covariance matrix $\mathrm{C}_{x v}$ is non-diagonal. External terms were omitted in $\mathbf{S}^{+}$, and simulations were done either with the non-diagonal form of $\mathrm{C}_{x v}$, or the $\mathrm{C}_{x v}$ was diagonalized by setting the offdiagonal terms to zero.

Computation of $\mathbf{S}^{+}$requires inversion of $\mathbf{S}^{T} \mathbf{S}$, which may be ill-conditioned. The inversion can be successfully completed by regularization. But, it was found that the standard regularization procedures (SVD truncation and Tikhonov regularizations) cannot simultaneously maintain low sensor noise and large SSS interference attenuation. To avoid this problem, we have removed from $\mathbf{S}$ all basis vectors, one at a time, and each time re-computed the condition number of the $\mathbf{S}^{T} \mathbf{S}$ based on the remaining vectors. We then removed the basis vector which reduced the condition number most. The procedure was repeated until the condition number was less than a specified value. Such procedure maintains specified span of singular values and yet reduces the SSS sensor noise and maintains large SSS interference attenuation. The vectors which were removed from the matrix $\mathbf{S}$ either have low amplitudes or are only slightly different from a linear combination of other vectors. Removal of these vectors will not result in a loss of important spatial topographies, at least within the accuracy of the specified span of singular values.

The SSS is known to attenuate the sensor noise, especially if the condition number of matrix $\mathbf{S}^{T} \mathbf{S}$ is small. We have adjusted the condition number limit to $10^{5}$, because at this value the sensor noise attenuation by the SSS for a reasonable range of spherical harmonic expansion orders is $\approx 1$.

We report results only on pseudo- $t$ and $f$ dual state beamformer constructs (Vrba and Robinson 2001), but statistically normalized (Barnes and Hillebrand 2003) and event related (Robinson 2004; Cheyne et al. 2007) beamformers were also simulated and exhibit similar behaviour.

\section{Description of Simulations}

A realistic, helmet shaped sensor array with 306 triple sensors (102 radial magnetometers and 204 planar gradiometers with $1.7 \mathrm{~cm}$ baseline) was simulated with random sensor gain error of $0.1 \%$. The spontaneous brain activity, the "brain noise", was modeled by 5000 dipole sources, present in all the following simulations, randomly distributed in a shell bounded by concentric spherical surfaces with 5 and $8 \mathrm{~cm}$ radii. Dipoles had random orientations and random amplitudes. After the brain noise simulation was completed, the rms brain noise density over all samples and all channels was normalized to $14 \mathrm{fT} / \sqrt{ } \mathrm{Hz}$ for planar gradiometers, which resulted in $33.8 \mathrm{fT} / \sqrt{ } \mathrm{Hz}$ noise density for magnetometers.

Either one or two tangential target sources were placed into the model sphere. Single source was positioned at $(0,0, a)$ and the two sources were positioned at $(0, \pm d / 2$, $\left.\left(a^{2}-d^{2} / 4\right)^{0.5}\right)$, where $a$ is the source distance from the model sphere center and $d$ is the source separation. Parameters used were $a=3,5,7,9 \mathrm{~cm}$ and $d=0.5,1$, $2 \mathrm{~cm}$, and source orientations were $(1,0,0)$. The duration of the time series was $T=100 \mathrm{~s}$, sample rate $f_{s}=150 \mathrm{~Hz}$, there were 156 triggers associated with the simulated source activity, and 0.2 -s pre- and 0.2 -s post-trigger intervals.

Source magnitudes were adjusted for signal-to-noise ratio (SNR) measured relative to the brain noise of $\mathrm{SNR}=0.1,1$, and 10 , with SNR defined as weighted averages $\left(M_{m} S N R_{m}+M_{p} S N R_{p}\right) /\left(M_{m}+M_{p}\right)$, where $M_{m}$ and $M_{p}$ are the numbers of magnetometer and planar gradiometer channels, and $S N R_{m}$ and $S N R_{p}$ are the magnetometer and planar gradiometer SNRs. The SNRs were defined as $\mathrm{SNR}=q^{2}|\mathbf{L}|^{2} /\left(v_{r m s}^{2} M\right)$, where $q$ is source magnitude, $\mathbf{L}$ is lead field vector (sensor response to a unit source), $v_{r m s}$ is nominal brain noise, and $M$ is the number of magnetometer or planar gradiometer channels.

For single source, the signal was $25 \mathrm{~ms}$ wide generalized Lorentzian peak with $50 \mathrm{~ms}$ latency relative to triggers, and 30\% random amplitude variation. When two sources were used, the signal of the first was the same as that of the single source, and the signal of the second was $40 \mathrm{~ms}$ wide peak with $90 \mathrm{~ms}$ latency and $40 \%$ random amplitude variation.

To assess the spatial resolution of the beamformer, its output peak dimensions were measured for single source simulations. The beamformer scan covered a volume which contained the source. Number of voxels, in which the beamformer output was larger than $1 / 2$ the associated peak amplitude, was counted. The peak volume was obtained by multiplying this count by voxel volume, and the peak dimension was approximated as a cube root of the peak volume. Voxels were kept sufficiently small such that at least 50 voxels were counted within the peak volume.

\section{Results}

Comparison of conventional and SSS beamformer peak dimensions for single source as a function of expansion order for different values of $a$ are shown in Fig. 1 for diagonalized instrumental noise covariance matrix and in 
Fig. 2 for non-diagonal noise covariance matrix. The conventional beamformers do not depend on the expansion order and their values are shown as horizontal lines spanning all expansion orders (gray solid and dashed lines).

Direct comparison of peak dimensions of conventional and SSS beamformers with diagonalized and non-diagonal noise covariance matrices is shown in Fig. 3a for dual state $t$ beamformer and $a=5 \mathrm{~cm}$. Similar comparison for the dual state $f$ beamformer would show that for non-diagonal
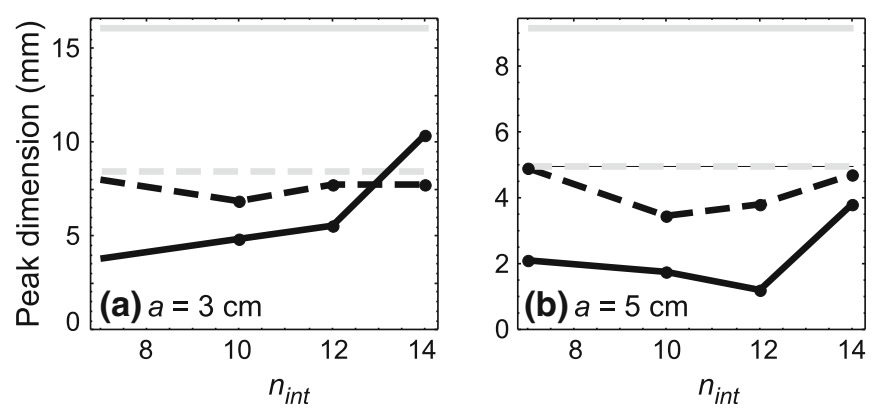

Fig. 1 Dependence of dual state beamformer peak dimension on internal expansion order, $n_{\text {int }}\left(n_{\text {ext }}=3\right)$. Diagonalized noise covariance matrix, $S N R=1$, parameter $a$ is the source distance from the
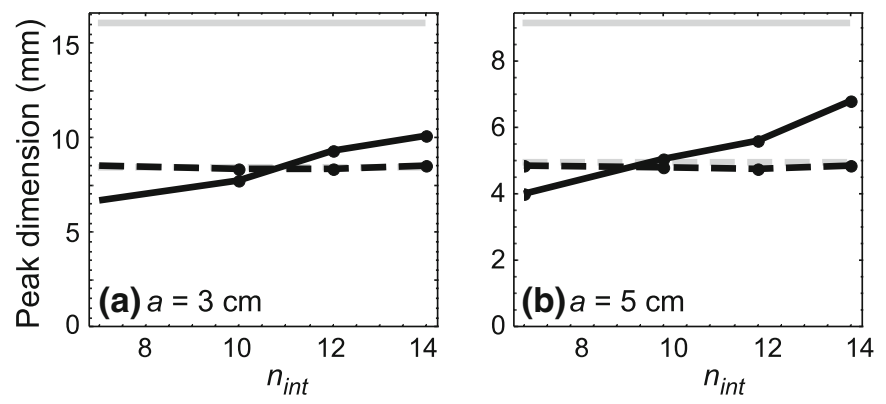

Fig. 2 Dependence of dual state beamformer peak dimension on internal expansion order, $n_{\text {int }}\left(n_{\text {ext }}=3\right)$. Non-diagonal noise covariance matrix, $S N R=1$, parameter $a$ is the source distance from the noise covariance matrix, the SSS and conventional peak dimensions are practically identical for all $n_{\text {int }}$; for the diagonalized noise covariance matrix the peak dimensions are the same for $n_{i n t}=7$ and 14, and the SSS peak dimension is slightly smaller than the conventional one for intermediate values $7<n_{\text {int }}<14$.

Actual shapes of the dual state beamformer $t$ responses to single source are shown in Figs. 3b-f as contour maps corresponding to intersection of the beamformer scan with
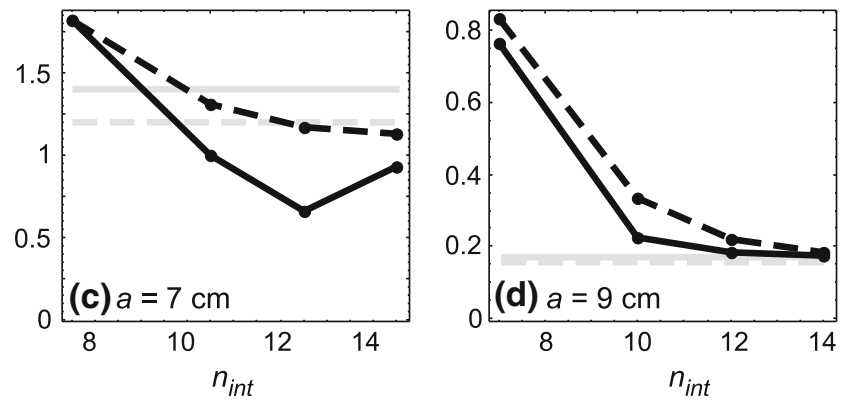

model center. Solid gray-Conventional, $t$; dashed gray-conventional, $f$; solid black-SSS, $t$; dashed black-SSS, $f$
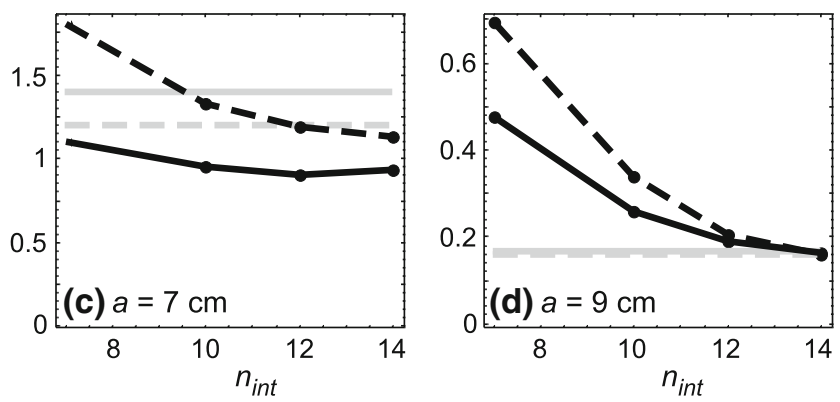

model center. Solid gray-Conventional, $t$; dashed gray-conventional, $f$; solid black-SSS, $t$; dashed black-SSS, $f$. Dashed black and gray lines for $a=3$ and $5 \mathrm{~cm}$ are nearly identical
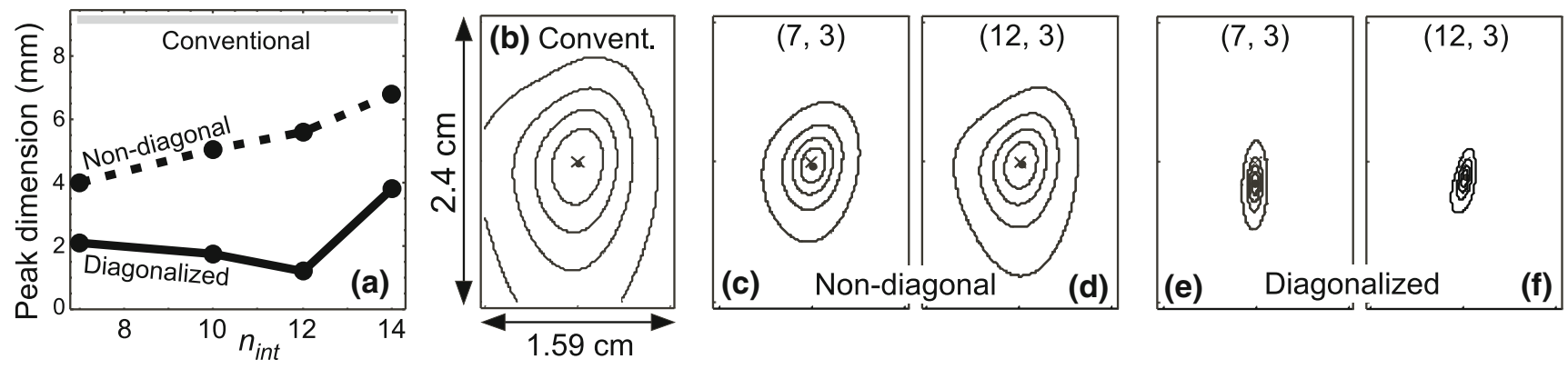

Fig. 3 Dependence of dual state beamformer $t$-peak dimension on beamformer type. $S N R=1, a=5 \mathrm{~cm}$. a Peak dimension as a function of $n_{\text {int }}\left(n_{\text {ext }}=3\right)$, solid gray-conventional; solid blackSSS, noise covariance matrix diagonalized; dashed black-SSS, nondiagonal noise covariance matrix. b-f Intersection of beamformer peak with $\mathrm{y}-\mathrm{z}$ plane, crosses indicate true source positions; b Conventional; c, d SSS, nondiagonal noise covariance matrix; e, f SSS, diagonalized noise covariance matrix; c, e SSS $\left(n_{\text {int }}, n_{\text {ext }}\right)=(7$, $3)$; d, f SSS $\left(n_{\text {int }}, n_{\text {ext }}\right)=(12,3)$ 

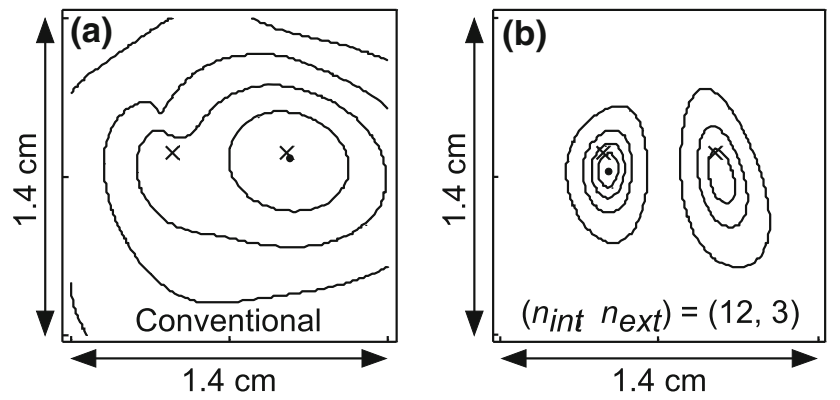

Fig. 4 Resolution of two sources separated by $0.5 \mathrm{~cm}$ at $a=5 \mathrm{~cm}$, $S N R=1.7$, dual state beamformers, $t$. Crosses indicate true source positions. a Conventional; b SSS $\left(n_{\text {int }}, n_{\text {ext }}\right)=(12,3)$ with diagonalized noise covariance matrix

$\mathrm{y}-\mathrm{z}$ plane passing through the voxel of maximum beamformer response. Figure $3 \mathrm{~b}$ corresponds to conventional beamformer and it exhibits the broadest peak. The peak dimensions of the SSS beamformer with non-diagonal noise covariance matrix are shown in Fig. 3c, d. The peak dimensions are smaller than those of the conventional beamformer in Fig. 3b, and the peak dimension increases with increasing expansion order, as also shown by dashed line in Fig. 3a. The peak dimensions of the SSS beamformer with diagonalized noise covariance matrix are shown in Fig. 3e, f. In this case, the SSS peaks are much sharper than that of the conventional beamformer in Fig. 3b, and in accordance with the solid line in Fig. 3a, the peak dimension of the expansion order $\left(n_{i n t}, n_{\text {ext }}\right)=(12,3)$ is slightly smaller than that of the expansion order $(7,3)$. Examples for the dual state $f$ beamformer are not shown, but the peak contours for the conventional and the SSS beamformers are nearly the same (see also Figs. 1 and 2).

The beamformer spatial resolution depends on data. To verify that the high spatial resolution of the dual state $t$ SSS beamformer is also maintained with more sources present, the response contours for two sources separated by $0.5 \mathrm{~cm}$ are shown in Fig. 4 for conventional and SSS beamformers. The SSS beamformer again exhibits significantly sharper spatial resolution. Similar behavior was also found for source separations of $0.5,1$, and $2 \mathrm{~cm}$ and for lower and higher SNRs than that shown in Fig. 4.

Simulations also show that the results for sensor gain errors of 0 and $0.1 \%$ are the same, the SSS beamformer outputs are only weakly dependent on the condition limit imposed on the $\mathbf{S}^{T} \mathbf{S}$ matrix, and the SNR related to the brain noise has the expected strong effect on spatial resolution (larger SNR results in better spatial resolution), but the character of the behaviour for all SNRs is similar to what was reported here for $\mathrm{SNR}=1$.

Simulations were also carried out with single state, statistically normalized, and event related conventional and SSS beamformers. Beamformer constructs which depend on power or power normalized by projected noise (single state $P$, dual state $Z^{2}$, differential $Z^{2}$, and event related beamformers) behave similarly to the dual state $t$ beamformer. Beamformer constructs which depend on ratio of powers or ratio of various power combinations (statistically normalized beamformer, either $t$ or probability $p$ ) behave similarly to the dual state $f$ beamformer.

\section{Discussion and Conclusions}

Spatial resolution of dual state SSS $t$-beamformers is significantly better than that of the conventional beamformers for deep sources and low expansion orders (solid lines in Figs. 1a, b and 2a, b). For deep sources, the SSS beamformer peak dimension increases with increasing expansion order. For very superficial sources, the spatial resolution of the SSS beamformer is roughly the same as that of the conventional beamformer if the expansion order is sufficiently high (Figs. 1d, 2d) and slightly worse if the expansion order is low (but still only about $0.5 \mathrm{~mm}$ ); the peak dimension monotonically decreases with increasing expansion order and attains the spatial resolution of the conventional beamformer for expansion order $n_{\text {int }}=14$. For intermediate source depths in Fig. 1c the SSS beamformer resolution is better than that of the conventional beamformer for $n_{i n t} \geq 10$, and in Fig. 2c for all expansion orders. Improvement of the SSS beamformer resolution for deep sources is larger for diagonalized than for non-diagonal noise covariance matrix.

Mechanism of the beamformer spatial resolution in the presence of brain noise is complex. We speculate that the improvement of the spatial resolution by the SSS beamformer for the deep sources may have the following origins: First, it is observed that the angle between signal space vectors corresponding to two deep sources is larger when calculated in the SSS expansion basis than when calculated in sensor space. For superficial sources, this difference diminishes. Second, if the expansion order is lower than necessary for superficial brain noise sources, but adequate for deeper target source, then the deep target sources will be described well by the SSS expansion, but the more superficial brain noise sources will not. Such "spatial low-pass filtering" of the superficial brain noise sources will alter their signal space vectors by decreasing their amplitudes and smoothing the corresponding field distributions. All these effects will improve spatial resolution of the beamformer.

Behavior is quite different for the dual state $f$ beamformers and deep sources. There the difference between the SSS and conventional beamformer resolutions is small for diagonalized noise covariance matrix (dashed lines in Fig. 1a, b), and resolutions are almost identical for non-diagonal noise 
covariance matrix (Fig. 2a, b). For superficial sources, the character of the SSS $f$ beamformers is similar to that of the $t$ beamformers, the conventional and SSS resolutions are the same only if the SSS expansion order is high. Lack of significant spatial resolution improvement for deep sources by the SSS $f$-beamformers is possibly caused by cancellation when forming power ratios.

The diagonalization of the noise covariance matrix significantly improves the SSS $t$-beamformer resolution. This is illustrated in Fig. 3a. The SSS beamformer with both non-diagonal and diagonalized noise covariance matrices exhibit sharper peaks than the conventional beamformer, but the peaks with the diagonalized noise covariance matrix are significantly sharper than those with non-diagonal matrix. Reasons for this behavior are presently under investigation.

The beamformer peak contours in Fig. 3b-f explicitly illustrate the spatial resolution improvement by the SSS type $t$ beamformers. For non-diagonal covariance matrix, the improvement is modest (compare Fig. 3c, d with b) and the peak dimension increases with increasing expansion order, as predicted by the dashed line in Fig. 3a. For diagonalized noise covariance matrix in Fig. $3 \mathrm{e}$, f the SSS peak size reduction over that of the conventional beamformer is dramatic.

Volume or dimension of a single peak was used as a measure of spatial resolution and was systematically investigated. Another possible measure of the spatial resolution is a distance between two sources at which the sources are resolved. This measure of the spatial resolution has not been investigated. However, it was shown that the better spatial resolution of the SSS beamformers is maintained even when two sources are present in the system, as shown in Fig. 4. Similar to the conventional beamformers, the spatial resolution of two sources by the SSS beamformers will depend on the source SNR, depth, and orientation.

Beamformers already are spatial filters which are sensitive to a target source and suppress contributions from other sources with forward solutions which do not match the target forward solution. In addition, the SSS $t$ beamformers can be adjusted to exhibit increased resolution for deeper sources. This increased resolution is achieved by lowering the expansion order to match the source depth. At the same time the lower expansion order will make the beamformer mismatched for superficial sources and will attenuate them. This mechanism effectively acts as an additional spatial filter which can be tuned to enhance deep sources and attenuate superficial sources.

Sensitivity of the SSS beamformers to the lead field inaccuracy has not been investigated. It could be speculated that the SSS beamformers would be less sensitive to the lead field inaccuracy because their dimensionality is lower. But this sensitivity will have to be established more rigorously by simulations.

In conclusion, beamformers were constructed which take advantage of the lesser number of the SSS expansion basis vectors than the physical MEG channels. The SSS beamformers perform as well as the conventional beamformers for superficial sources, provided that the expansion order is sufficiently high. But for deeper sources and the beamformer outputs which depend on power or power difference normalized by the projected noise, the SSS beamformers exhibit much better spatial resolution than the conventional beamformers. In addition, because the SSS beamformers operate on a lower dimensional system and do not require integration over the sensor area, the computational speed is increased.

Open Access This article is distributed under the terms of the Creative Commons Attribution Noncommercial License which permits any noncommercial use, distribution, and reproduction in any medium, provided the original author(s) and source are credited.

\section{References}

Ahonen AI, Hämäläinen MS, Ilmoniemi RJ, Kajola MJ, Knuutila JET, Simola JT, Vilkman VA (1993) Sampling theory for neuromagnetic detector arrays. IEEE Trans Biomed Eng 40:859869

Barnes GR, Hillebrand A (2003) Statistical flattening of MEG beamformer images. Hum Brain Mapp 18:1-12

Cheyne D, Bostan AC, Gaetz W, Pang EW (2007) Event-related beamforming: a robust method for presurgical functional mapping using MEG. Clin Neurophys 118:1691-1704

Gross J, Kujala J, Hamalainen M, Timmermann L, Schnitzler A, Salmelin R (2001) Dynamic imaging of coherent sources: studying neural interactions in the human brain. Proc Natl Acad Sci USA 98:694-699

Nenonen J, Taulu S (2005) Signal space separation. In: Yoshimine T (ed) MEG source estimation. Proceedings of 20th annual meeting of Japan biomagnetism and biomagnetic society. J Jpn Biomagn Biomagn Soc 18:14-15

Robinson SE (2004) Localization of event-related activity by SAM(erf). In: Halgren E et al (eds) Biomag 2004. Biomag 2004 Ltd, Boston, pp 583-584

Robinson S, Vrba J (1998) Functional neuroimaging by synthetic aperture magnetometry. In: Yoshimoto $\mathrm{T}$ et al (eds) Recent advances in biomagnetism. Tohoku University Press, Sendai, Japan, pp 302-305

Sekihara K, Nagarajan S, Poeppel D, Marantz A (2004) Asymptotic SNR of scalar and vector minimum-variance beamformers for neuromagnetic source reconstruction. IEEE Trans Biomed Eng 51:1726-1734

Taulu S, Kajola M (2005) Presentation of electromagnetic multichannel data: the signal space separation method. J Appl Phys 97(12):97-106

Van Veen BD, Van Drongelen W, Yuchtman M, Suzuki A (1997) Localization of brain electrical activity via linearly constrained minimum variance spatial filtering. IEEE Trans Biomed Eng 44:867-880

Vrba J, Robinson SE (2001) Signal processing in MEG. Methods 25:249-271 